\title{
Situation Socio-Demographique, Culturelle Et Comportement Nutritionnel Des Femmes Enceintes En Consultation Prenatale Au Chu De Cocody-Abidjan (Cote d'Ivoire)
}

\author{
Essé Sonia-Estelle, \\ Yeboué Kouamé Hermann, \\ Amoikon Kouakou Ernest,
}

Laboratoire de Nutrition et Pharmacologie, UFR Biosciences, Université Félix Houphouet-Boigny, Abidjan, Côte d'Ivoire.

Doi: 10.19044/esj.2018.v14n18p288 URL:http://dx.doi.org/10.19044/esj.2018.v14n18p288

\begin{abstract}
The objective of this work is to evaluate the socio-demographic and cultural status, and the nutritional behavior of pregnant women received in medical consultation at the Gynecology-Obstetrics Department of the University Hospital Center of Cocody-Abidjan (Côte d'Ivoire). A survey was conducted on a cohort of 504 pregnant women attending antenatal clinics in the period from August 26, 2015 to February 23, 2017. The survey method consisted of collecting information on socio-demographic, cultural and nutritional status, pregnant women, from a survey card. The results showed that these women, whose age varies between 15 and 44 years, are predominantly Ivorian, with $49.23 \%$ of them belonging to the Akan ethnic group. Burkinabe women represent more than half of non-Ivorian women. The Christian women represent $68.25 \%$, against $29.76 \%$ of Muslim women. Of those surveyed, $82.14 \%$ are single, $82.54 \%$ are literate, compared to $17.86 \%$ married and $17.46 \%$ illiterate. Living conditions have revealed that 3-room apartment buildings are the most inhabited, and many of them enjoy the amenities of a modern home. At the nutritional level, $1.19 \%$ of respondents followed a diet recommended by a doctor and $31.35 \%$ have dietary prohibitions. $0.99 \%$ of this study population regularly consume alcohol. Food groups such as breads, cereals, starchy foods and pulses are the most consumed (68.06 \%); meat is more consumed than fish. Fresh fruits and vegetables are less present in the dietary habits of these respondents. From this study, it could be concluded that the respondents have a satisfactory sociodemographic situation. However, civil marriage is not a proof of marital stability. The percentage of divorced could give an indication of this stability,
\end{abstract}


although in Côte d'Ivoire, customary marriage is the most recognized by the community. Finally, in Côte d'Ivoire, eating habits are often known. But it is a good and balanced diet that the population often ignores.

Keywords: Pregnant women, socio-demographic and cultural situation, nutritional behavior, Côte d'Ivoire

\section{Resume}

L’objectif de ce travail est d'évaluer le statut socio-démographique et culturel, et le comportement nutritionnel des femmes enceintes reçues en consultation médicale au service de gynécologie-obstétrique du Centre Hospitalier Universitaire de Cocody-Abidjan (Côte d'Ivoire). Une enquête a porté sur une cohorte de 504 femmes enceintes venues en consultation prénatale dans la période du 26 Août 2015 au 23 Février 2017. La méthode d'enquête consistait à collecter des informations portant sur le statut sociodémographique, culturel et le comportement alimentaire des femmes enceintes, à partir d'une fiche d'enquête. Les résultats ont montré que ces femmes, dont 1'âge varie entre 15 et 44 ans, sont à majorité Ivoiriennes, avec 49,23 \% d'entre elles appartenant au groupe ethnique Akan. Les Burkinabées représentent plus de la moitié des non-Ivoiriennes. Les chrétiennes représentent 68,25\%, contre 29,76\% de musulmanes. Parmi ces enquêtées, $82,14 \%$ sont célibataires, $82,54 \%$ sont lettrées, contre 17,86 \% de mariées et $17,46 \%$ d'analphabètes. Les conditions de vie ont révélé que les habitations de type "appartement à 3 pièces sont les plus habitées, et beaucoup d'entre elles bénéficient des commodités d'une habitation moderne. Au niveau nutritionnel, $1,19 \%$ des enquêtées ont suivi un régime alimentaire recommandé par un médecin et $31,35 \%$ ont des interdits alimentaires. 0,99\% de cette population d'étude consomment régulièrement de l'alcool. Les groupes d'aliments comme les pains, céréales, féculents et légumes secs sont les plus consommés $(68,06 \%)$; la viande est plus consommée que le poisson. Les fruits et légumes frais sont moins présents dans les habitudes alimentaires de ces enquêtées. De cette étude, on pourrait conclure que les enquêtées ont une situation sociodémographique satisfaisante. Cependant, le mariage civil n'est pas une preuve de stabilité matrimoniale. Le pourcentage de divorcées pourrait donner une indication sur cette stabilité, encore qu'en Côte d' Ivoire, c'est le mariage coutumier qui est le plus reconnu par la communauté. Enfin, en Côte d'Ivoire, les habitudes alimentaires sont souvent connues. Mais c'est une alimentation bonne et équilibrée que la population ignore souvent.

Mots-clés : Femmes enceintes, situation socio-démographique et culturelle, comportement nutritionnel, Côte d'Ivoire 


\section{Introduction}

La grossesse ou gestation est un processus physiologique au cours duquel la progéniture vivante d'une femme se développe dans son corps, depuis la conception jusqu'à ce qu'elle puisse survivre ou jusqu'à son interruption par un avortement artificiel ou naturel (fausse couche), hors du corps de la mère (Nathan, 2008). La grossesse constitue l'une des étapes les plus importantes dans la vie d'une femme. En effet, durant neuf mois, l'organisme de la femme enceinte subit une série de modifications liées au développement du fœetus, à la croissance des tissus maternels, au maintien de l'homéostasie maternelle et à la préparation à l'allaitement (King, 2000). La maternité sans risque, les problèmes de santé maternelle et infantile ont toujours été les préoccupations majeures des décideurs politiques, des partenaires du développement et des services de soins de santé. Leur but collectif est de réduire la mortalité maternelle et infantile, et les complications liées à l'accouchement qui constituent un réel problème de santé publique dans la plupart des pays en développement (Zakaria et Laribick, 2014). La mortalité maternelle demeure un fléau qui touche durement les pays en développement, et particulièrement l'Afrique où les conditions socio démographiques et culturelles, économiques et sanitaires précaires exposent la femme aux complications de la grossesse et de l'accouchement. Selon les statistiques de l'OMS, environ 830 femmes meurent chaque jour dans le monde pendant la grossesse ou l'accouchement ou dans le post-partum immédiat (OMS, 2016).

La Côte d'Ivoire fait partie des pays africains qui enregistrent un nombre important de décès dû aux complications liées à la grossesse ou à l'accouchement, avec un rapport de mortalité maternelle estimé à 614 décès pour 100000 naissances vivantes (EDS-MICS, 2011-2012). En plus de ces conditions de vie défavorables, il a été aussi démontré qu'une nutrition adéquate est essentielle pendant la grossesse et est considérée comme un facteur déterminant pour le déroulement harmonieux de la grossesse et de l'accouchement (Pellaë, 2001) ; autrement dit pour la santé de la mère, la croissance et le développement du fotus (Kaiser et al., 2008 ; Blumfield et al., 2013 ; Brenseke et al., 2013). Pendant cette période particulière, la femme enceinte doit, en effet, couvrir les besoins nutritionnels accrus de son organisme ainsi que ceux de l'enfant à naître (Girard, 1993 ; Simon, 2001). Dans l'optique d'avoir des données récentes et fiables sur la typologie des femmes enceintes, une enquête visant à évaluer la situation sociodémographique et culturelle et le comportement alimentaire des femmes enceintes, a été réalisée au service de gynécologie-obstétrique du Centre Hospitalier Universitaire (CHU) de Cocody-Abidjan (Côte d'Ivoire). 


\section{Méthodologie}

\section{Cadre de l'étude et population cible}

Abidjan est une ville qui s'étend sur une superficie de $422 \mathrm{~km}^{2}$. Elle est située au Sud-Est de la Côte d'Ivoire. C'est la capitale économique Ivoirienne et également la ville la plus peuplée de l'Afrique de l'Ouest Francophone. Le recensement général de la population et de l'habitat (RGPH) de 2014 a estimé la population de la ville d'Abidjan à 4707404 habitants (RGPH, 2014), soit plus de $20 \%$ de la population Ivoirienne. Elle compte 10 communes réparties en Abidjan Nord et Abidjan Sud situés respectivement au Nord et au Sud de la lagune Ebrié, et 4 sous-préfectures. La commune de Cocody, réputée pour ses quartiers résidentiels et ses grandes institutions, avec 447055 habitants (RGPH, 2014), abrite un Centre Hospitalier Universitaire (CHU). Le CHU de Cocody comprend 13 services dont le service de gynécologie-obstétrique où s'est déroulée une enquête dans la période du 26 Août 2015 au 23 Février 2017. La population cible était constituée de 504 femmes enceintes (toutes nationalités confondues), âgées de 15 à 44 ans, venues en consultation au sein de ce service. Etaient exclues, toutes femmes non enceintes.

\section{Méthodes d'enquête}

Une fiche d'enquête conçue spécialement pour cette étude nous a permis de collecter des informations sur les conditions environnementales, le cadre de vie et le comportement nutritionnel des enquêtées. Cette fiche d'enquête comporte une série de questionnaires auxquelles devraient répondre les femmes enceintes. Ces questions renseignent sur la situation sociodémographique et culturelle dont l'âge, la nationalité, la religion, la situation matrimoniale et le type de foyer, le niveau d'instruction, le type d'habitation et les commodités (nombre de pièces habitées, présence d'électricité, d'eau de robinet, type de latrines).

La fiche d'enquête comprend aussi un volet alimentaire. Il s'agit de régime alimentaire particulier pendant la période de grossesse, les interdits alimentaires et la fréquence de consommation des différents groupes d'aliments. Ceci a donné les fréquences suivantes : «jamais » qui veut dire que cet aliment n'est pas consommé par les enquêtées; « très rare » qui signifie une consommation du groupe de l'aliment une fois par mois au plus ; « rare » au cas où l'aliment est consommé une fois chaque 2 semaines, la fréquence « très peu » où la consommation se fait une à 2 fois par semaine ; «peu » pour une fréquence de consommation allant de 3 à 4 fois par semaine et enfin « beaucoup » qui signifie que l'aliment est consommé au moins une fois par jour. 


\section{Traitement statistique}

Les logiciels Sphinx version 5. et IBM SPSS statistics 20.0 ont été utilisés pour la saisie et le traitement des données. Les graphiques ont été réalisés à l'aide de « Excel 2013 ».

\section{Résultats}

\section{Situation socio-demographique et culturelle}

Les femmes en grossesse reçues en consultation au service de gynécologie-obstétrique du CHU de Cocody ont été réparties en quatre classes d'âge. Celles dont l'intervalle d'âge est compris entre 30 et 39 ans sont en majorité, avec un taux de 47,62 \%, suivi des femmes âgées de 20 et 29 ans $(41,67 \%)$. La classe d'âge inférieur ou égal à 19 ans représente 7,14 \% et enfin $3,57 \%$ pour les sujets âgés de 40 ans et plus (Figure 1). L'âge moyen des enquêtées est de 29,7 ans, avec un minimum de 15 ans et un maximum de 44 ans.

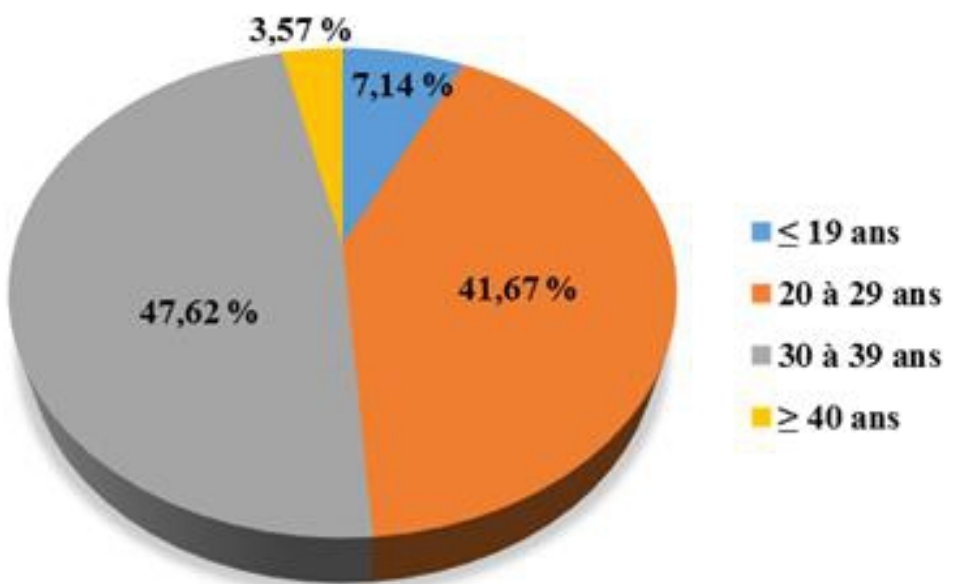

Figure 1 : Répartition des femmes enceintes selon l'âge

Concernant l'origine des femmes enceintes, la nationalité Ivoirienne est classée majoritaire avec un pourcentage de $89,88 \%$ (Figure 2) et le groupe Akan a le plus fort taux $(49,23 \%)$ parmi les quatre grands groupes ethniques de la Côte d'Ivoire (Figure 3). Parmi les 10,12 \% de femmes gestantes de nationalité étrangères, les Burkinabées représentent 50,98 \%, soit un peu plus de la moitié. 


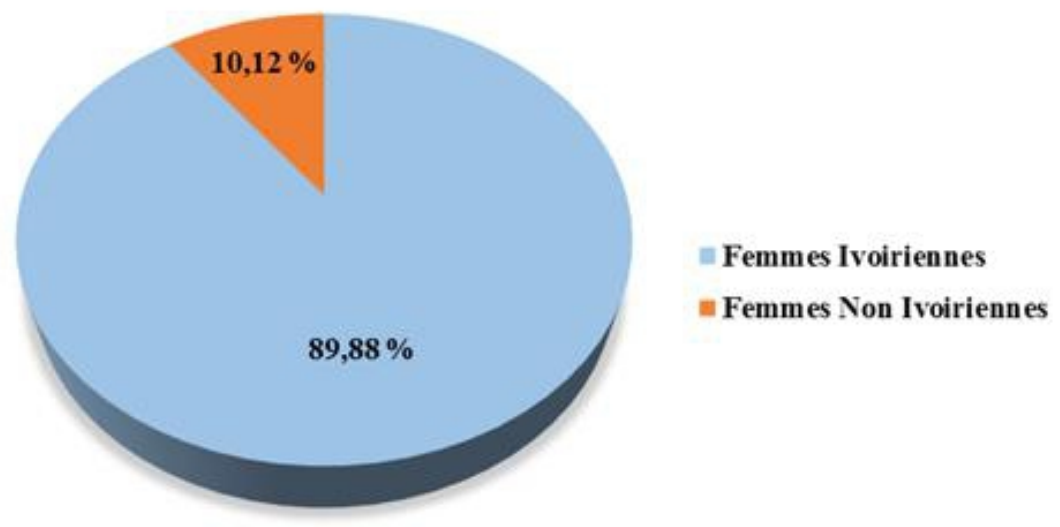

Figure 2 : Répartition des femmes enceintes selon la nationalité

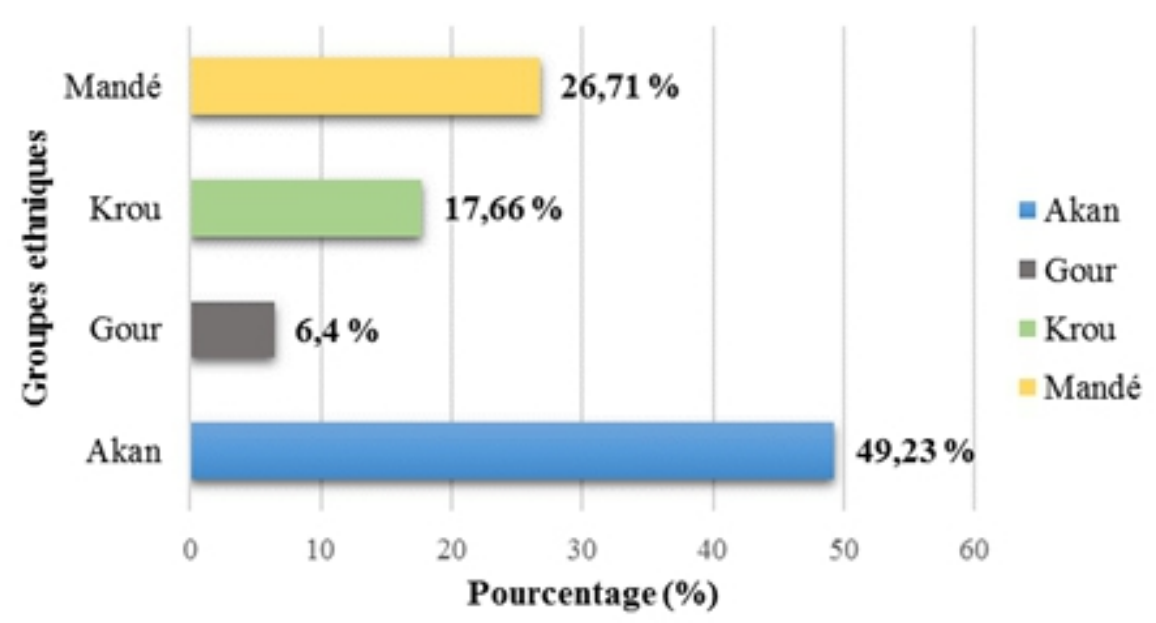

Figure 3 : Répartition des femmes enceintes selon le groupe ethnique

La plupart des femmes qui ont participé à cette étude pratiquent la religion chrétienne $(68,25) \%$ contre $29,76 \%$ de musulmanes. Les autres, c'est-à-dire les animistes, bouddhistes etc... sont seulement estimées à 1,98 \% (Figure 4). 


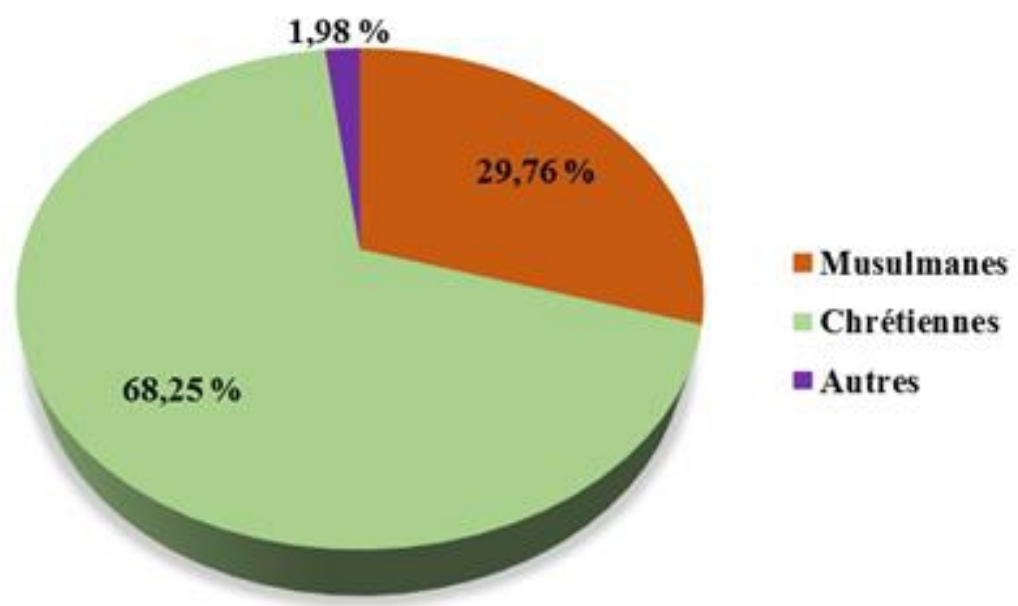

Figure 4 : Répartition des femmes enceintes selon la religion

Pour ce qui est de la situation matrimoniale et le type de foyer dans lequel vivent les enquêtées, seulement 17,86 \% sont mariées contre 82,14\% de célibataires (Figure 5). Toutes femmes ayant effectué ou non le mariage traditionnel ou religieux mais pas le mariage civil sont considérées comme des femmes célibataires. Ces femmes vivent, soit en concubinage, soit seules ou toujours en famille. 54,17\% de ces enquêtées vivent seules ou en famille. Parmi les 45,83 \% vivant dans un foyer, $8,73 \%$ sont des foyers de type polygamique, c'est-à-dire qu'elles ont des co-épouses et les $37,1 \%$ restant sont dans des foyers monogamiques (Figure 6).

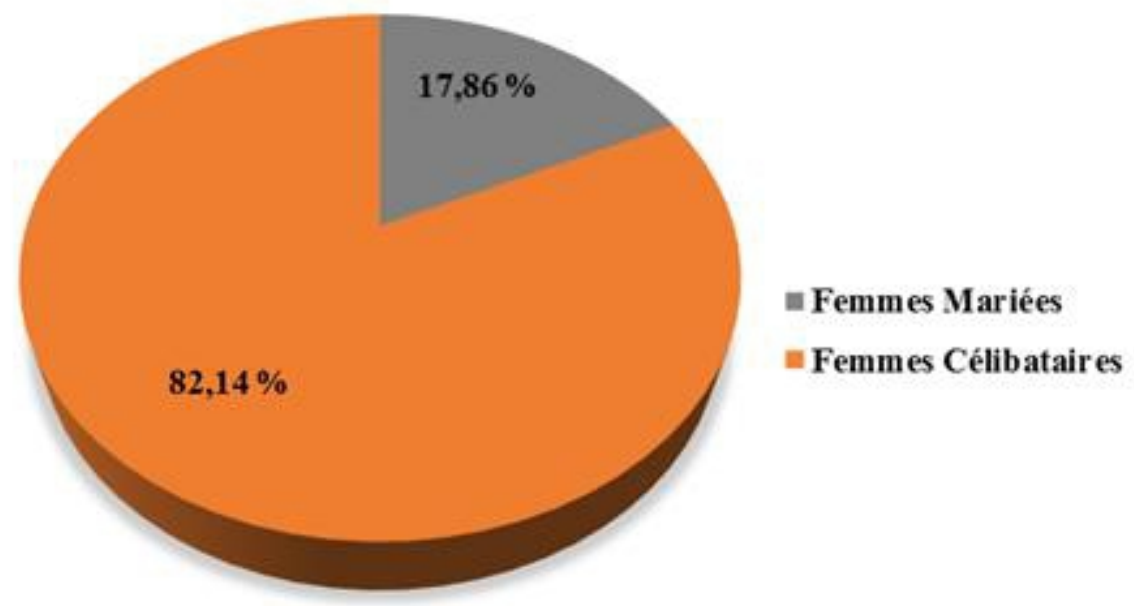

Figure 5 : Répartition des femmes enceintes selon la situation matrimoniale 


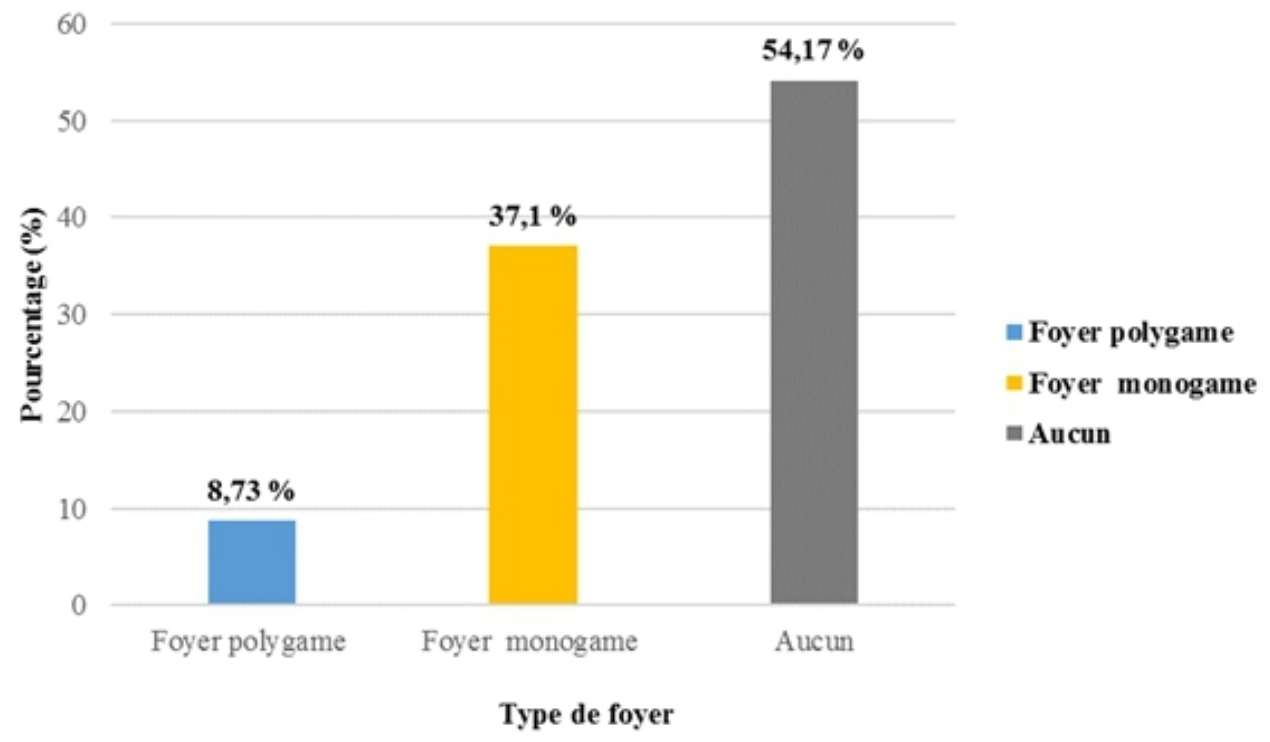

Figure 6: Répartition des femmes enceintes selon le type de foyer

Le taux de femmes gestantes n'ayant jamais été scolarisées, donc analphabètes, est estimé à 17,46\% \% Les autres ont été scolarisées, avec des niveaux scolaires répartis comme suit : 18,25\% de niveau primaire; 30,56\% de niveau secondaire et enfin 33,73\% de niveau supérieur (Figure 7).

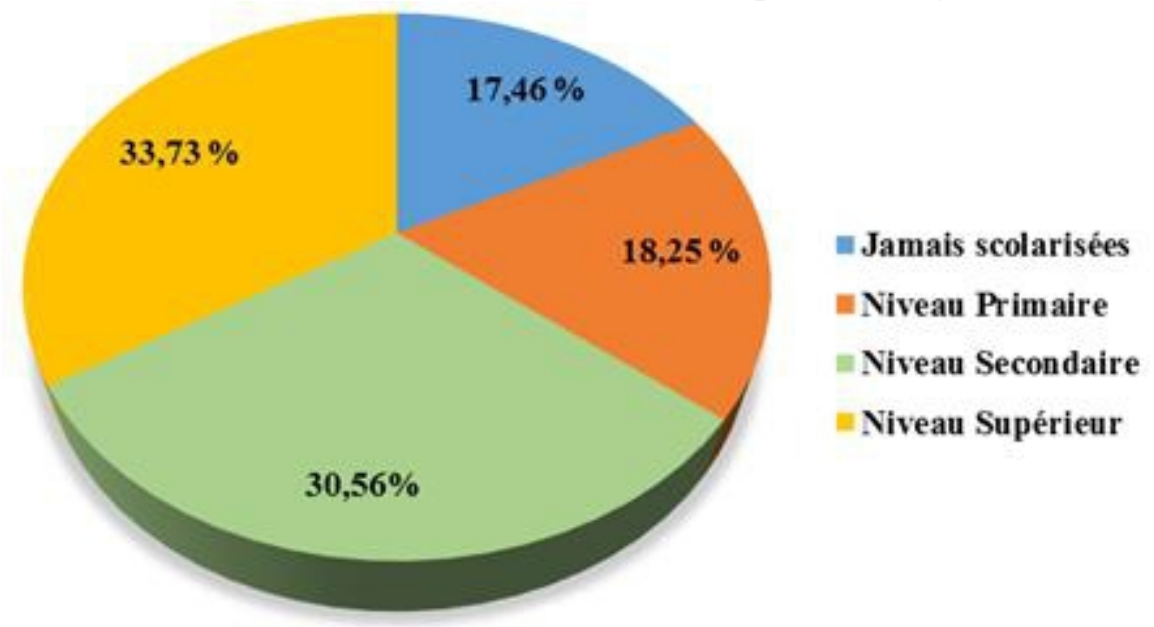

Figure 7 : Répartition des femmes enceintes selon le niveau de scolarisation 
Les conditions dans lesquelles vivent les femmes enceintes constituent l'un des aspects socio-culturels les plus importants. Ce sont entre autres, le type d'habitation, le nombre de pièces de ces habitations et les commodités qui s'y trouvent, comme la présence d'électricité, d'eau de robinet, de latrine et le type de latrines. Ainsi, les appartements sont les plus habités avec $48,81 \%$; suivis de $36,11 \%$ pour les maisons de type villa. $11,71 \%$ vivent dans les habitats précaires et enfin 3,37 \% dans les habitations de type traditionnel (Figure 8).
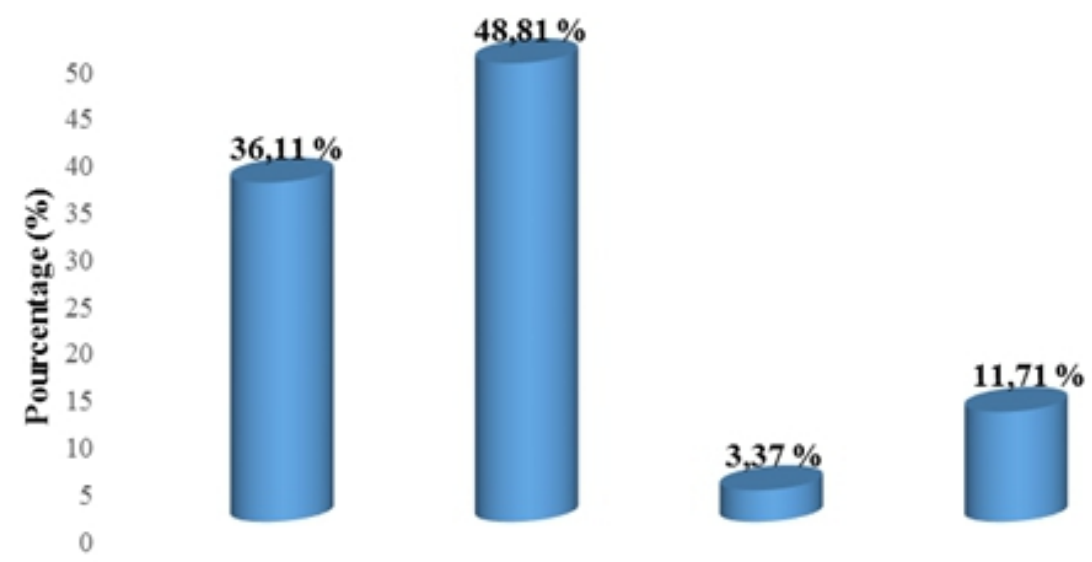

Villa

Appartement

Type traditionnel

Habitat précaire

Type d'habitat

Figure 8: Répartition des femmes enceintes selon le type d'habitat

Pour ce qui est du nombre de pièces qui composent ces habitations, $46,03 \%$ de ces femmes habitent des maisons à deux chambres et salon (3 pièces) et le plus faible taux $(9,33 \%)$ revient à celles occupant des maisons à 4 pièces (Figure 9).
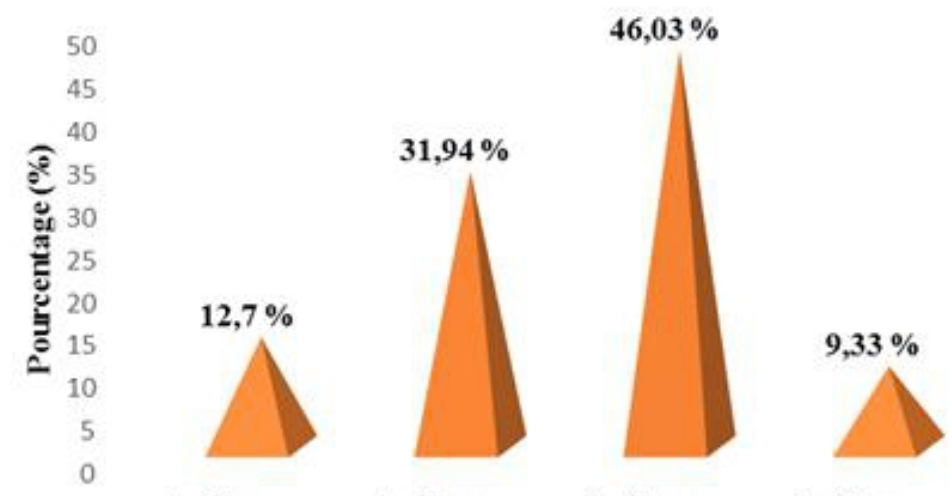

1 pièce

2 pièces

3 pièces

4 pièces

\section{Nombre de pièces}

Figure 9: Répartition des femmes enceintes selon le nombre de pièces de l'habitat 
Presque toutes les enquêtées bénéficient de l'installation d'électricité. En effet, seules 0,99 \% habitent des maisons non électrifiées (Figure 10). En ce qui concerne l'eau de robinet, 93,65 \% des femmes enceintes en consomment, contre 6,35 \% qui n'y ont pas accès (Figure 11). 99,21 \% des enquêtées bénéficient de la présence de latrine. Celles qui utilisent des latines de types moderne représentent $91 \%$ de la population d'étude, contre $9 \%$ qui se contentent des latrines de type traditionnel (Figure 12).

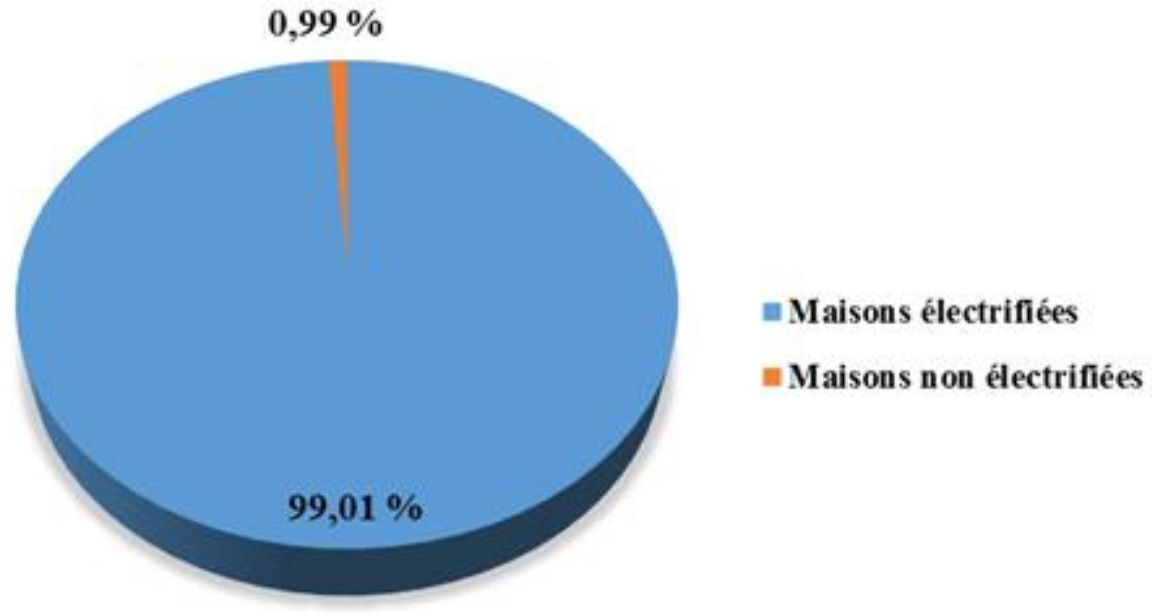

Figure 10 : Présence ou non d'électricité dans l'habitat

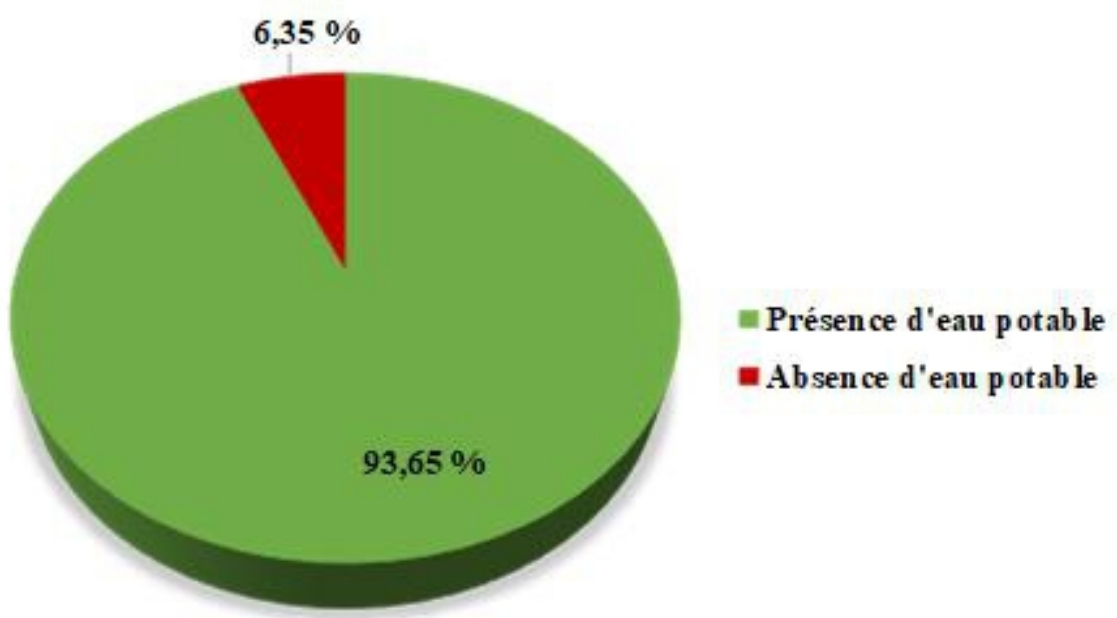

Figure 11 : Présence ou non d'eau de robinet dans le ménage 


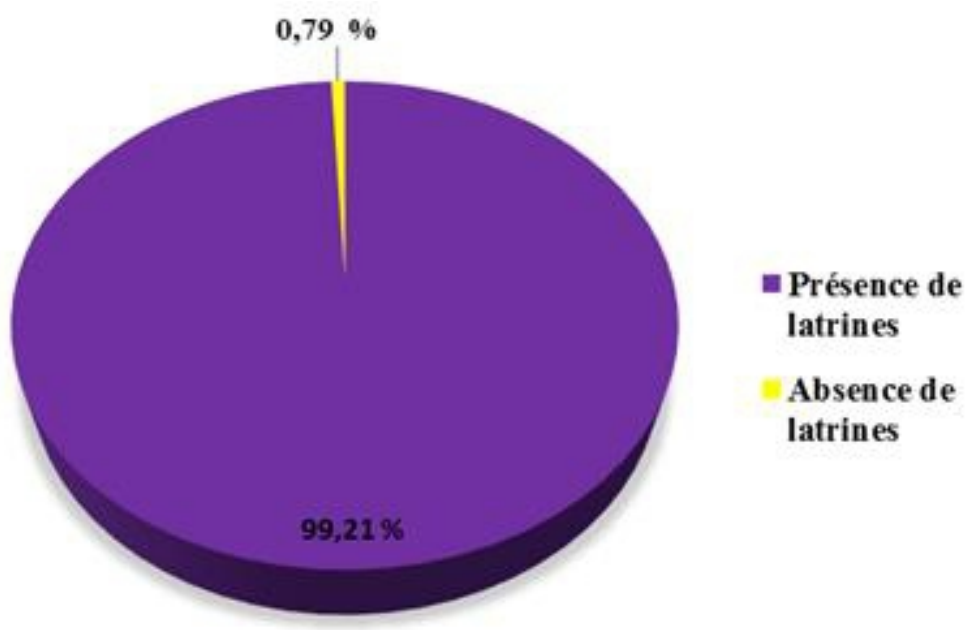

Figure 12 : Présence ou non de latrines dans les habitats

\section{Comportement nutritionnel}

Certaines femmes suivent un régime alimentaire particulier pour des raisons de santé, ou tout simplement, pour éviter les excès en certains nutriments. Ces femmes ont été évaluées à $1,19 \%$ selon l'enquête. Cependant, 98,81\% de ces enquêtées ne suivent aucun régime particulier (Figure 13).

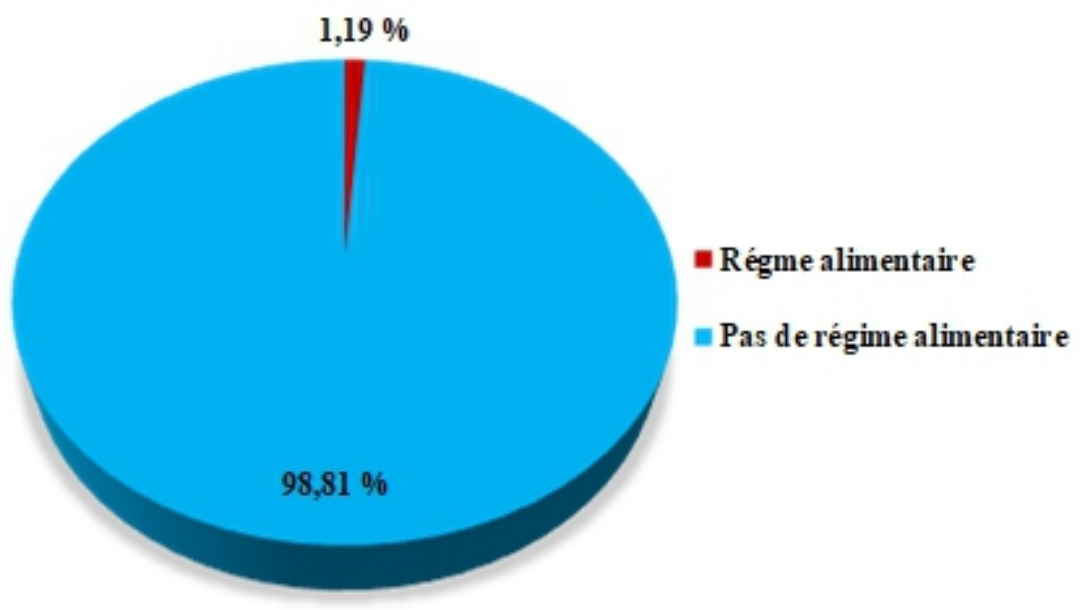

Figure 13 : Répartition des femmes enceintes selon un régime alimentaire particulier

Selon les coutumes et traditions africaines, ou encore les pratiques religieuses, 31,35\% des femmes enceintes ont des interdits alimentaires, contre $68,65 \%$ qui consomment toutes sortes d'aliments sans aucune interdiction (Figure 14). 


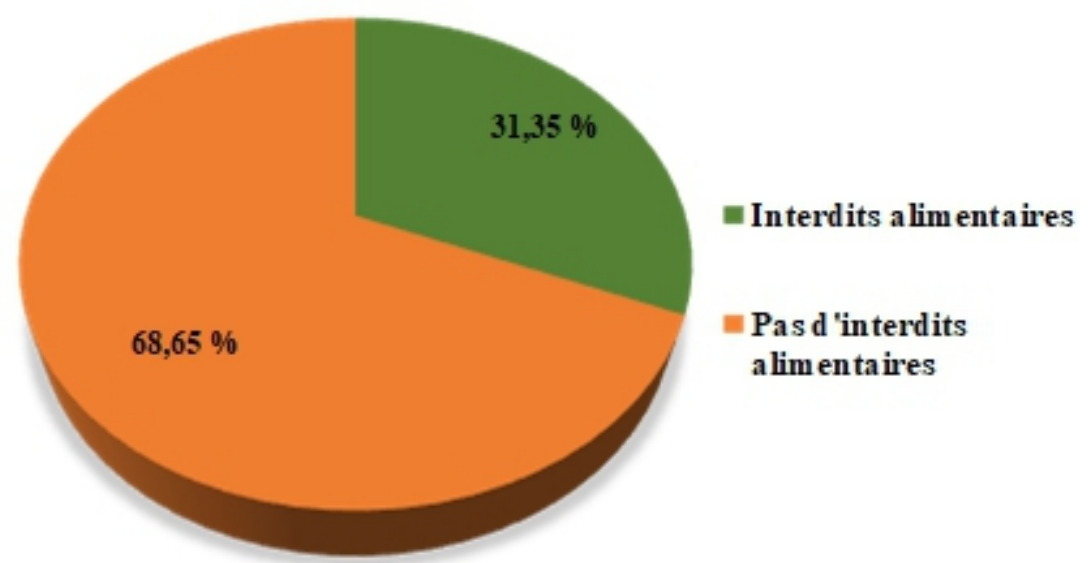

Figure 14 : Répartition des femmes enceintes selon les interdits alimentaire

Après les enquêtes, les résultats ont montré que presque toutes les femmes enceintes s'hydratent fréquemment, et en grand nombre, en consommant beaucoup d'eau. $15,28 \%$ de ces femmes consomment de l'alcool, et $0,99 \%$ en consomment régulièrement. Les fruits et légumes frais ne font pas partie de leurs habitudes alimentaires. En effet, jusqu'à 68,06 \% des femmes en mangent très rarement, c'est-à-dire une fois par mois. Les deux groupes d'aliments que sont : les pains, céréales, féculents, légumes secs et viandes, poissons, œufs sont beaucoup consommés, avec des taux respectifs de $45,44 \%$ et $44,44 \%$. L'utilisation journalière de lait et produits laitiers dans l'alimentation de ces femmes est estimée à seulement $11,31 \%$; alors que $35,51 \%$ en consomment une fois par mois. Les matières grasses et produits sucrés font partie de la consommation journalière à un très faible taux, respectivement $5,75 \%$ et $7,14 \%$ (Tableau I).

Tableau I : Fréquence de consommation des groupes d'aliments par les enquêtées.

\begin{tabular}{|c|c|c|c|c|c|c|c|c|c|c|c|c|}
\hline \multirow[t]{2}{*}{ Groupes d'aliments } & \multicolumn{2}{|c|}{ Jamais } & \multicolumn{2}{|c|}{ Très rare } & \multicolumn{2}{|c|}{ Rare } & \multicolumn{2}{|c|}{ Très peu } & \multicolumn{2}{|c|}{ Peu } & \multicolumn{2}{|c|}{ Beaucoup } \\
\hline & Moy & $\%$ & Moy & $\%$ & Moy & $\%$ & Moy & $\%$ & Moy & $\%$ & Moy & $\%$ \\
\hline Eau & 00 & 00 & 00 & 00 & 01 & 0,20 & 20 & 3,97 & 91 & 18,05 & 392 & 77,78 \\
\hline Alcool & 427 & 84,72 & 50 & 9,92 & 14 & 2,78 & 08 & 1,59 & 05 & 0,99 & 00 & 00 \\
\hline Fruits et légumes frais & 00 & 00 & 343 & 68,06 & 35 & 6,94 & 33 & 6,55 & 40 & 7,94 & 53 & 10,51 \\
\hline $\begin{array}{c}\text { Pains, céréales, féculents } \\
\text { légumes secs }\end{array}$ & 00 & 00 & 108 & 21,43 & 49 & 9,72 & 49 & 9,72 & 69 & 13,69 & 229 & 45,44 \\
\hline Laits et produits laitiers & 00 & 00 & 179 & 35,51 & 95 & 18,85 & 84 & 16,67 & 89 & 17,66 & 57 & 11,31 \\
\hline $\begin{array}{l}\text { Viandes, poissons et } \\
\text { œufs }\end{array}$ & 00 & 00 & 116 & 23,02 & 54 & 10,71 & 49 & 9,72 & 61 & 12,10 & 224 & 44,44 \\
\hline Matières grasses & 00 & 00 & 306 & 60,71 & 61 & 12,10 & 48 & 9,52 & 60 & 11,90 & 29 & 5,75 \\
\hline Produits sucrés & 00 & 00 & 281 & 55,75 & 53 & 10,52 & 69 & 13,69 & 65 & 12,90 & 36 & 7,14 \\
\hline
\end{tabular}

\section{Discussion}

L'enquête réalisée sur les femmes enceintes venues en consultation prénatale au CHU de Cocody a permis de recueillir des informations sur leur situation socio-démographique et culturelle. La moyenne d'âge de ces 
enquêtées (29,7 ans) est la même que celle trouvée par Touati-Mecheri dans ses travaux à El Khroub en Algérie (Touati-Mecheri, 2011). L’âge moyen des enquêtées est sensiblement égale à 29,3 et 29,2 ans, lesquels correspondent respectivement aux résultats des enquêtes faites dans les PMI de la ville de Tébessa (Taleb et al., 2012) et chez les femmes enceintes Maghrébines vivant en Espagne (Roville-Sausse et al., 2001). La majorité des femmes enceintes de notre étude ont un âge compris entre 30 et 39 ans (47,62\%), alors qu'en Malawi, ce sont plutôt les femmes enceintes dont l'âge est compris entre 20 et 29 ans qui ont le taux le plus élevée avec 56,9 \% (Titilayo et al., 2016). Le moment idéal pour la maternité se situe entre 20 et 34 ans. Après 35 ans, la femme court un risque accru de présenter des complications au cours de la grossesse, telles que l'avortement spontané, l'hypertension artérielle, les hémorragies et la mort du fœtus (Bee et Boyd, 2003). A l'inverse, le jeune âge de l'adolescente, son immaturité biologique, affective et sociale aggravent les difficultés observées au cours de sa grossesse et de sa maternité. Le risque apparaît d'autant plus élevé que la mère est très jeune, avec une fréquence plus grande de l'hypertension artérielle, de la toxémie, de l'anémie, des difficultés d'accouchement, de la prématurité, de l'hypotrophie fœtale et des malformations (Haesevoets, 2008).

En ce qui concerne la religion, les chrétiennes représentent un taux extrêmement plus élevé que celui des musulmanes $(68,25 \%$ contre $29,76 \%)$; les $1,98 \%$ restant étant attribués aux animistes, bouddhistes etc. Ces résultats sont différents des enquêtes démographiques faites en Malawi qui ont donné $83,9 \%$ de chrétiennes, $14,8 \%$ de musulmanes et 1,3\% pour les autres (Titilayo et al., 2016). La situation matrimoniale et le type de foyer sont des éléments qui contribuent largement à l'épanouissement de la femme enceinte. Ainsi, cette étude a montré que seulement 17,86 \% des femmes sont mariées, contre $82,14 \%$ de célibataires. Ce faible taux de mariées pourrait s'expliquer par le fait que le mariage en Afrique est généralement basé sur le mariage traditionnel (c'est-à-dire la dote) ou religieux. Cependant, dans ce travail, seule la femme qui a contracté un mariage civil a été considérée comme femme mariée. Toutes les autres ont été classées dans le groupe des femmes célibataires. Parmi ces enquêtées, 8,73 \% vivent dans des foyers polygames. Ces résultats sont semblables à ceux des études menées à Ouagadougou (Burkina-Faso) sur les femmes enceintes qui ont donné $8 \%$ de femmes célibataires (Testot-Ferry, 2008). Le taux de femmes analphabètes s'élève à $17,46 \%$; ce taux est supérieur à celui trouvé dans l'enquête faite par TouatiMecheri en Algérie, qui est de 12,5\% (Touati-Mecheri, 2011). Les conditions d'habitation et le milieu dans lequel évolue le ménage sont des facteurs qui ont une influence sur l'état de santé de la population (MSPRH, 2004). Ainsi, le type d'habitation et les commodités dans lesquels vivent les parturientes pourraient avoir une influence sur l'évolution et le déroulement de la 
grossesse. Selon l'enquête, 11,71 \% vivent dans les habitats précaires, ce qui se rapproche des résultats trouvés en Constantine en Algérie correspondant à 10,1\% (Touati-Mecheri, 2011). Cependant, les habitats de type traditionnel sont en minorité $(3,37 \%)$. Les résultats de cette étude concernant les commodités dans les habitations sont comparés à ceux de Touati-Mecheri (2011) en Algérie. En effet, le CHU de cocody a enregistré des chiffres plus élevés que ceux de la ville de Constantine, au niveau des habitations à 3 pièces (46,03\% contre $40 \%$ ), de la présence d'électricité $(99,01 \%$ contre $96,9 \%)$ et d'eau de robinet $(93,65 \%$ contre $78,3 \%)$. Cependant, les latrines de type moderne ont donné un pourcentage moins élevé: 90,4\%, contre 93,3\% (Touati-Mecheri, 2011).

$\mathrm{Au}$ niveau du comportement nutritionnel, il a été remarqué que seulement $1,19 \%$ des parturientes ont suivi un régime alimentaire particulier. Cela serait dû à leur état de santé, parce qu'elles étaient souvent diabétiques ou hypertendues. A cela, s'ajoute les interdits alimentaires ; en effet, 31,35\% de ces femmes étaient confrontées à des interdits alimentaires pour des raisons religieuses ou tout simplement selon les lois de la tradition. Ces interdits alimentaires pourraient entraîner des carences en certains nutriments, et même aboutir à une malnutrition. La grossesse est un état physiologique exigeant en éléments nutritionnels. Mais, elle ne nécessite pas de régime particulier pour les femmes dont les apports étaient auparavant satisfaisants. Il convient toutefois d'éviter, à la fois, les insuffisances et les excès, et d'être attentive à certains besoins spécifiques (Perrin et Simon, 2002). Les femmes, qui n'ont pas une alimentation suffisante et suffisamment variée, sont plus exposées à des complications durant la grossesse et l'accouchement (FAO, 2000).

A partir des fréquences de consommation alimentaire, il a été établi les moyennes de consommation dans chaque groupe d'aliment. Ainsi, 68,06\% des enquêtées consomment très rarement les fruits et légumes frais. Elles ne respectent donc pas les règles de la Commission économique des Nations Unies pour l'Europe, le Secrétariat général de la Communauté du Pacifique, l'Institut International de Recherches sur les Politiques Alimentaires et la communauté mondiale qui prônent la consommation de 5 fruits et légumes par jour pour la santé (FAO/OMS, 2004). En effet, les fruits et légumes sont une composante importante d'une alimentation saine. Consommés quotidiennement en quantité suffisante, ils pourraient aider à prévenir des affections d'importance majeure, comme les maladies cardiovasculaires, certains cancers etc... Selon un Rapport sur la santé dans le monde en 2002, la faible consommation de fruits et légumes est la cause d'environ $31 \%$ des cardiopathies ischémiques et de $11 \%$ des accidents vasculaires cérébraux dans le monde (OMS, 2002). Les matières grasses et produits sucrés sont des aliments qui nécessitent une consommation avec modération, surtout pendant les périodes vulnérables comme celle de la grossesse. Cela serait bénéfique 
pour la mère et l'enfant qui naîtra. L'enquête a aussi révélé que seulement $5,75 \%$ et $7,14 \%$ consomment respectivement les aliments gras et sucrés chaque jour. 15,28\% de la population d'étude consomment l'alcool. Cette proportion représente moins de la moitié de la consommation d'alcool chez les femmes enceintes en Côte d'Ivoire (Yao et al., 2014). Cependant, elle est plus élevée que le taux de consommation d'alcool chez les femmes enceintes Canadiennes qui serait de 10,5\%, alors que 62,4 \% des femmes non enceintes en consomment (Chudley et al., 2005). Même si les recherches n'ont pas encore indiqué exactement le niveau de consommation à risque pour le foetus, il serait plus commode pour la femme enceinte de se priver d'alcool pendant la grossesse, car il est reconnu que la consommation d'alcool est la plus nocive des formes de pollution intra-utérine transmises par le comportement maternel (Abel et Sokol, 1987 ; Ebrahim et al., 1998).

\section{Conclusion}

Avec un intervalle d'âge allant de 15 à 44 ans, et une moyenne d'âge de 29,7 ans, notre population d'étude est pour la plupart dans la tranche d'âge de 30 à 39 ans. La religion chrétienne est pratiquée à 68,25\% et plus de 3/4 des femmes qui ont participé à cette enquête sont célibataires. Le taux d'analphabètes de cette population d'étude s'élève à $17,46 \%$, une campagne de sensibilisation sur le niveau d'instruction serait donc utile pour réduire ce taux. Après l'enquête, il a été constaté que seulement $1,19 \%$ des femmes ont suivi un régime alimentaire pendant leur grossesse, $31,35 \%$ ont des interdits alimentaires. Sur une cohorte de 504 femmes enceintes, seulement 10,51\% consomment au moins 5 fruits et légumes par jour. Les aliments gras et sucrés sont beaucoup consommés par moins de $10 \%$ des femmes et 15,28\% consomment l'alcool. Pendant la période de gestation, la femme devient vulnérable et est sujette aux maladies, aux infections et à la malnutrition. Certaines femmes courent un réel danger de santé si leur condition de vie est défavorable et qu'elles ne respectent pas le mode de consommation alimentaire. Cependant, il serait judicieux d'engager une politique de prévention et de sensibilisation des femmes en âge de procréer, et particulièrement les femmes enceintes, sur le mode de vie, l'alimentation, et surtout sur les dangers auxquels elles s'exposent en consommant l'alcool.

\section{References:}

1. Abel, E. L. \& Sokol, R. J. (1987). Incidence of fetal alcohol syndrome and economic impact of fas-related anomalies. Drug and Alcohol Dependence, 19: 51-70.

2. Bee, H. \& Boyd, D. (2003). Psychologie de développement des âges de la vie. $2^{\text {ème }}$ Edition de Boeck, 36-75. 
3. Blumfield, M. L., Hure, A. J., Macdonald-Wicks, L., Smith, R. \& Collins, C. E. (2013). A systematic review and meta-analysis of micronutrient intakes during pregnancy in developed countries. Nutrition Reviews, 71 (2): 118-132.

4. Brenseke, B., Prater, M. R., Bahamonde, J. \& Gutierrez, J-C. (2013). Current thoughts on maternal nutrition and fetal programming of the metabolic syndrome. Journal of Pregnancy, 2013: 1-13.

5. Chudley, A. E., Conry, J., Cook, J. L., Loock, C., Rosales, T. \& LeBlanc, N. (2005). Fetal alcohol spectrum disorder: Canadian guidelines for diagnosis. Canadian Medical Association Journal, 172 (5): 1-21.

6. Ebrahim, S. H., Luman, E. T., Floyd, R. L., Murphy, C. C., Bennett, E. M. \& Boyle, C. C. A. (1998). Alcohol consumption by pregnant women in the United States during 1988-1995. Obstetrics and Gynecology, 92: 187-192.

7. EDS-MICS (2011-2012). Etudes Démographiques et de la Santé et à Indicateurs Multiples de la Côte d'Ivoire. Rapport de synthèse, 20112012, 20 pages.

8. FAO (2000). L'état de l'insécurité alimentaire dans le monde. Food and Agriculture Organization, 31: 11-17.

9. FAO/ OMS (2004). Fruits et légumes pour la santé. Rapport de l'atelier commun FAO/OMS, Kobe (Japon), 55 pages.

10. Girard, J. (1993). Nutrition et croissance foetale. In : « Traité de nutrition pédiatrique »; Ricour C., Ghisolfi J., Putet G., Goulet O. Editions Maloine, 1088 pages, Paris: 295-311.

11. Haesevoets, Y. (2008). Traumatismes de l'enfance et de l'adolescence. Editions de Boeck, 151-335.

12. Kaiser, L. \& Allen, L. H. (2008). American Dietetic Association. Position of the American Dietetic Association: nutrition and lifestyle for a healthy pregnancy outcome. Journal of the American Dietetic Association, 108: 553-561.

13. King J-C. (2000). Physiology of pregnancy and nutrient metabolism. The American Journal of Clinical Nutrition, 71 (5): 1218-1225.

14. Ministère de la Santé de la Population et de la Réforme Hospitalière (MSPRH) (2004). Enquête nationale sur la santé de la famille, Algérie 2002. Rapport principal, 374 pages.

15. OMS (2002). Rapport sur la santé dans le monde - Réduire les risques et promouvoir une vie saine, Genève, Organisation mondiale de la Santé, 2002.

16. Nathan (2008). Savignac, B., Meslier, F., Delaunay, C. \& Oustalniol, J. Guide d'anatomie et de physiologie, formations paramédicales. Nathan, 288 pages. 
17. OMS (2016). Mortalité maternelle; Aide-mémoire $n^{\circ} 348$; Novembre 2016.

18. Pellaë, M. (2001). La lettre de l'Institut Danone $\mathrm{n}^{\circ}$ 55: Poids et grossesse : importance du statut nutritionnel antérieur, 6 pages.

19. Perrin, A. E. \& Simon, C. (2002). Nutrition de la femme enceinte. Cahier de Nutrition et de Diététique, 37 (1): 59-64.

20. RGPGH (2014). Résultats globaux des districts, régions, départements et sous-préfectures. Secrétariat technique permanent du comité technique du Recensement Général de la Population et de l'Habitat. Côte d'Ivoire, 26 pages.

21. Roville-Sausse, F., Truc, J-B. \& Jacob, D. (2001). Gain de poids maternel durant la grossesse dans certaines communautés vivant en France. Revue d'Epidémiologie et de Santé Publique, 49: 439-447.

22. Simon, C. (2001). Nutrition de la femme enceinte et allaitant. In : «Traité de nutrition clinique de l'adulte. (Basdevant A. Laville M. Lerebours éd.).»p283- 292. Médecine-Sciences Flammarion, 723 pages, Paris.

23. Taleb, S., Kaib, M. \& Deghboudj, N. (2012). Evaluation de l'état nutritionnel des femmes enceintes fréquentant les PMI de la ville de Tebessa (Algérie). Biosanté, 11: 9-16.

24. Testot-Ferry, A. (2005). Les comportements alimentaires des femmes enceintes à Ouagadougou, (Burkina Faso). Mémoire du Diplôme d'Etudes Supérieures Spécialisées. Nutrition et Alimentation dans les Pays en Développement. Université de Montpellier II, 33 pages.

25. Titilayo, A., Palamuleni1, M. E. \& Omisakin, O. (2016). Sociodemographic factors influencing adherence to antenatal iron supplementation recommendations among pregnant women in malawi: analysis of data from the 2010. Malawi demographic and health survey. Malawi Medical Journal, 28 (1): 1-5.

26. Touati-Mecheri, D. (2011). Statut nutritionnel et sociodémographique d'une cohorte de femmes enceintes d'El Khroub (Constantine, Algérie). Répercussions sur le poids de naissance du nouveau-né (année 2002). Mémoire de thèse de Doctorat en Sciences alimentaire, 2011. INATAA, Université de Mentouri de Constantine, 220 pages.

27. Yao, K. M., Assi, B. D., Bâ, A., Adou, K. F. J-B. \& Tako, N. A. (2014). Epidémiologie de la consommation d'alcool par les femmes enceintes en Côte d'Ivoire : enquête sur 834 cas à Abidjan. Journal of Applied Biosciences, 80: 7024-7030.

28. Zakaria, H. \& Laribick, D. B. (2014). Socio-economic determinants of dietary diversity among women of child bearing ages in northern Ghana. Food Science And Quality Management, 34: 12-25. 